\title{
A COMPARATIVE ANALYSIS OF SUSTAINABILITY REPORTS PUBlisheD BY GLOBAL ENTERPRISES: STANDALONE CSR VERSUS INTEGRATED REPORTING
}

\author{
Mert Demir and Maung K. Min
}

The BRC Academy Journal of Business 11, no. 1 (2021): 23-51.

https://dx.doi.org/10.15239/j.brcacadjb.2020.11.01.ja02

\section{WEB APPENDIX}

https://dx.doi.org/10.15239/j.brcacadjb.2021.11.01.wa02 
Figure 1 - CSR reporting trends

\section{CSR Reporting Trends}

2017 calendar year

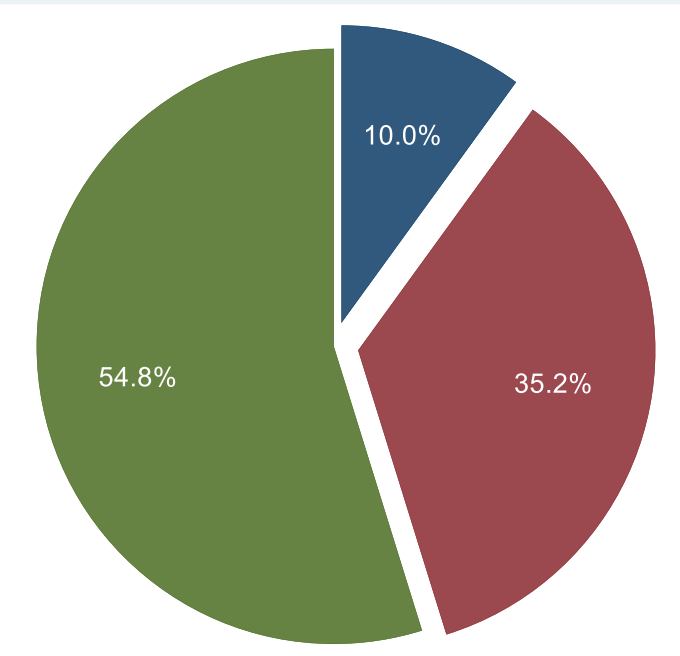

\begin{tabular}{|ll|}
\hline Integrated & No Report \\
Standalone & \\
\hline
\end{tabular}

Source: www.csrsmonitor.org 
Figure 2 - Regional differences in reporting trends

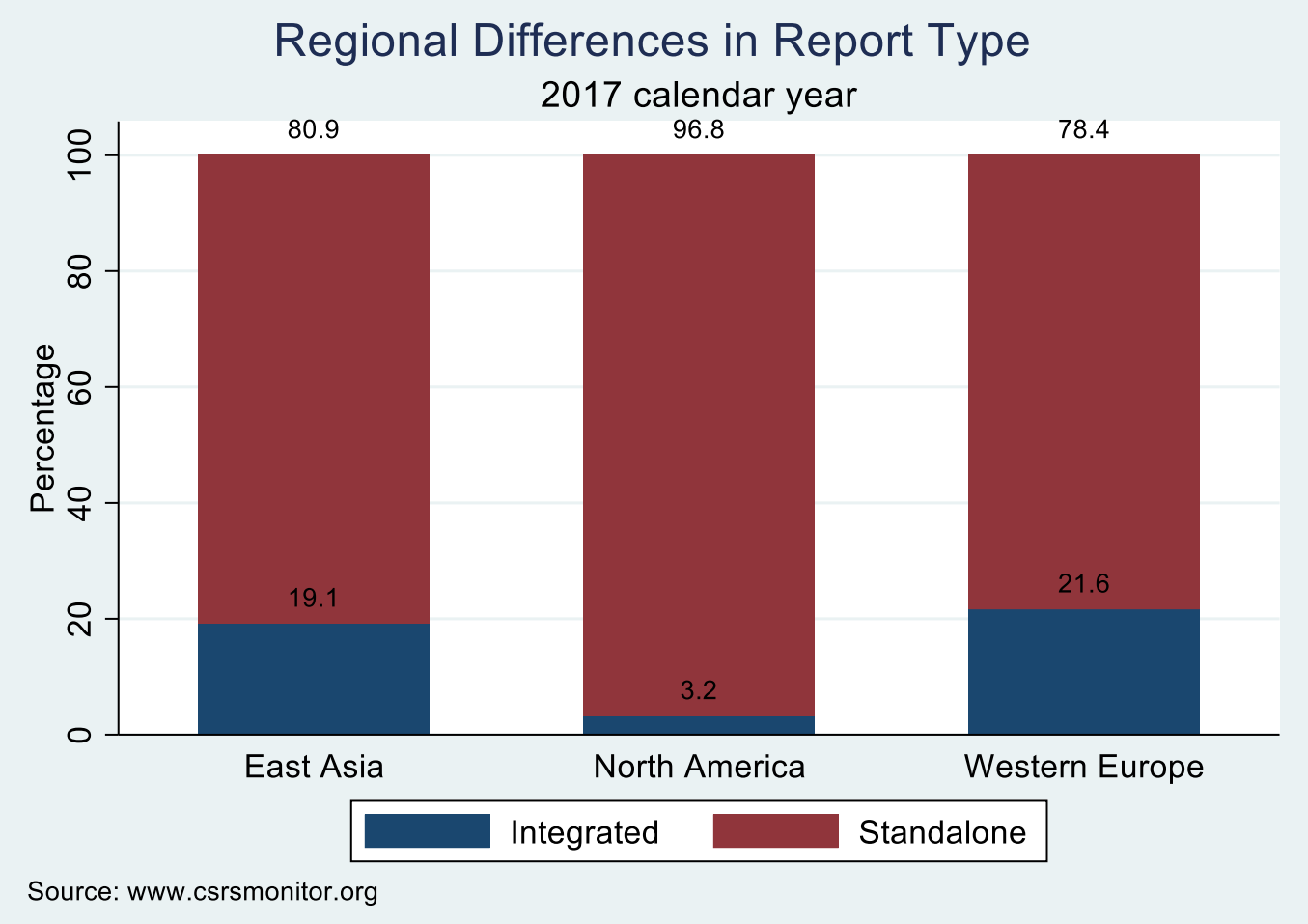


Figure 3 - The Monitor's average CSR reporting transparency scores across regions

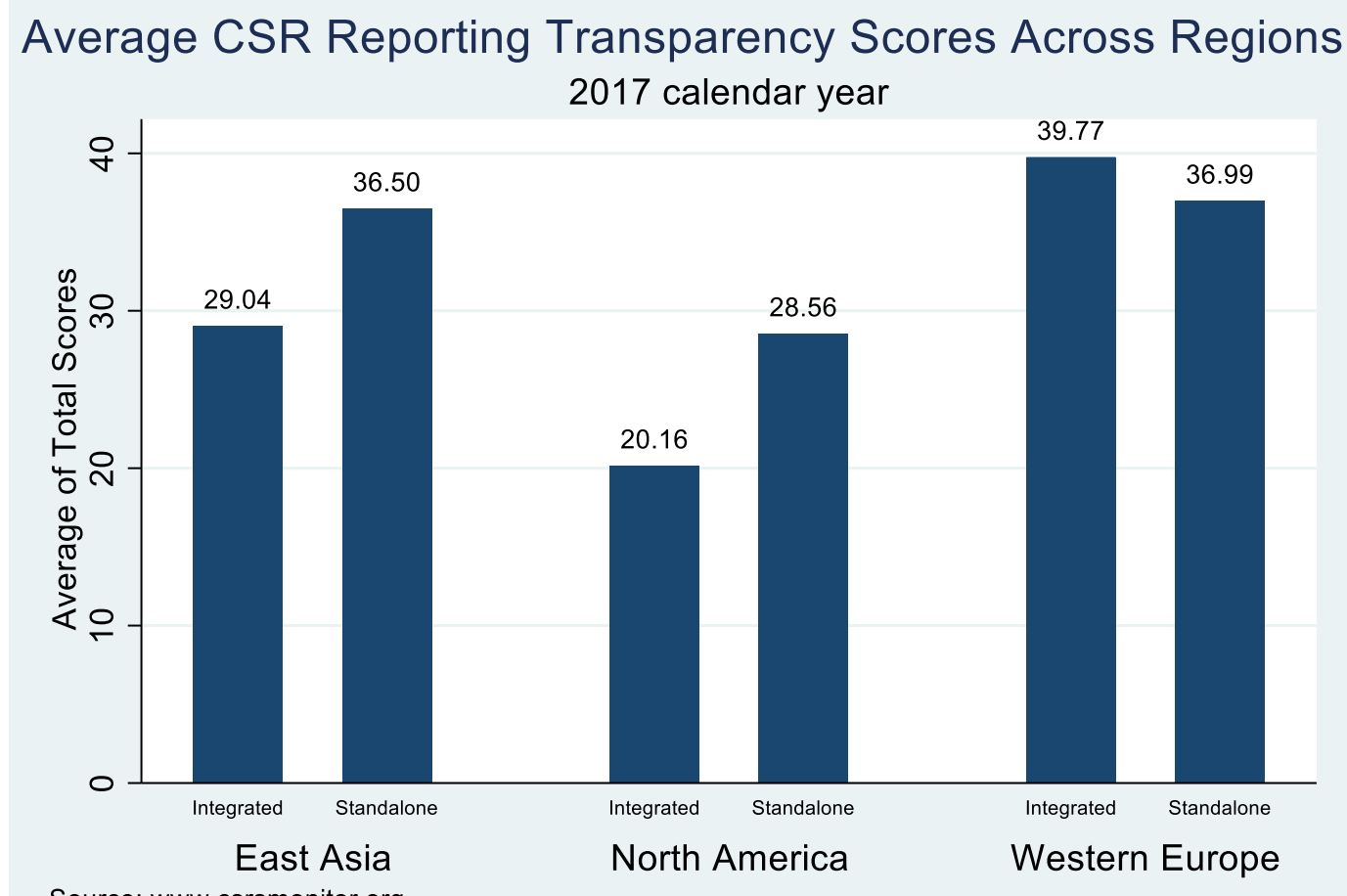


Table 1 - Tests of differences in average scores between integrated and standalone reports

\begin{tabular}{lll|ll|rc} 
& \multicolumn{2}{c|}{ Integrated } & \multicolumn{2}{c|}{ Standalone } & & \\
\cline { 2 - 7 } & $\mathrm{N}$ & Mean & $\mathrm{N}$ & Mean & Difference & $\mathrm{p}$-value \\
\hline Chair's Message & 50 & 55.91 & 274 & 50.36 & 5.54 & 0.01 \\
Environment & 50 & 31.10 & 274 & 38.25 & -7.14 & 0.02 \\
Philanthropy & 50 & 48.53 & 274 & 50.49 & -1.95 & 0.56 \\
Stakeholder Engagement & 50 & 24.71 & 274 & 31.20 & -6.49 & 0.02 \\
Supply Chain Management & 50 & 25.53 & 274 & 34.79 & -9.26 & 0.01 \\
Labor Relations & 50 & 36.16 & 274 & 40.76 & -4.60 & 0.17 \\
Governance & 50 & 69.40 & 274 & 26.35 & 43.05 & 0.00 \\
Anti-corruption & 50 & 29.80 & 274 & 26.14 & 3.66 & 0.39 \\
Human Rights & 50 & 24.94 & 274 & 33.49 & -8.55 & 0.04 \\
Codes of Conduct & 50 & 16.13 & 274 & 19.87 & -3.74 & 0.06 \\
Integrity Assurance & 50 & 22.00 & 274 & 24.21 & -2.21 & 0.61 \\
\hline Equal-weighted Total & 50 & 34.93 & 274 & 34.17 & 0.75 & 0.69 \\
Total excluding Governance & 50 & 31.48 & 274 & 34.96 & -3.48 & 0.08
\end{tabular}


Table 2 - Tests of differences in average scores between integrated and standalone reports: North America

\begin{tabular}{|c|c|c|c|c|c|c|}
\hline & \multicolumn{2}{|c|}{ Integrated } & \multicolumn{2}{|c|}{ Standalone } & \multirow[b]{2}{*}{ Difference } & \multirow[b]{2}{*}{$p$-value } \\
\hline & $\mathrm{N}$ & Mean & $\mathrm{N}$ & Mean & & \\
\hline Chair's Message & 3 & 66.67 & 91 & 48.85 & 17.82 & 0.01 \\
\hline Environment & 3 & 10.37 & 91 & 37.05 & -26.68 & 0.00 \\
\hline Philanthropy & 3 & 55.56 & 91 & 48.35 & 7.21 & 0.73 \\
\hline Stakeholder Engagement & 3 & 4.76 & 91 & 25.67 & -20.90 & 0.03 \\
\hline Supply Chain Management & 3 & 17.65 & 91 & 33.94 & -16.29 & 0.20 \\
\hline Labor Relations & 3 & 13.07 & 91 & 33.66 & -20.58 & 0.02 \\
\hline Governance & 3 & 40.00 & 91 & 16.92 & 23.08 & 0.38 \\
\hline Anti-corruption & 3 & 0.00 & 91 & 14.87 & -14.87 & 0.00 \\
\hline Human Rights & 3 & 9.80 & 91 & 26.89 & -17.09 & 0.22 \\
\hline Codes of Conduct & 3 & 3.89 & 91 & 17.29 & -13.40 & 0.04 \\
\hline Integrity Assurance & 3 & 0.00 & 91 & 10.62 & -10.62 & 0.00 \\
\hline Equal-weighted Total & 3 & 20.16 & 91 & 28.56 & -8.39 & 0.12 \\
\hline Total excluding Governance & 3 & 18.18 & 91 & 29.72 & -11.54 & 0.08 \\
\hline
\end{tabular}


Table 3 - Tests of differences in average scores between integrated and standalone reports: Western Europe

\begin{tabular}{lll|ll|cc} 
& \multicolumn{2}{c|}{ Integrated } & \multicolumn{2}{c}{ Standalone } \\
\cline { 2 - 7 } & $\mathrm{N}$ & Mean & $\mathrm{N}$ & Mean & Difference & $\mathrm{p}$-value \\
\hline Chair's Message & 24 & 53.79 & 87 & 49.37 & 4.42 & 0.17 \\
Environment & 24 & 40.52 & 87 & 44.54 & -4.01 & 0.30 \\
Philanthropy & 24 & 49.44 & 87 & 47.97 & 1.47 & 0.74 \\
Stakeholder Engagement & 24 & 26.64 & 87 & 32.76 & -6.12 & 0.09 \\
Supply Chain Management & 24 & 30.88 & 87 & 37.32 & -6.44 & 0.23 \\
Labor Relations & 24 & 42.89 & 87 & 43.95 & -1.06 & 0.83 \\
Governance & 24 & 76.25 & 87 & 26.44 & 49.81 & 0.00 \\
Anti-corruption & 24 & 42.92 & 87 & 32.07 & 10.85 & 0.11 \\
Human Rights & 24 & 24.02 & 87 & 37.05 & -13.03 & 0.04 \\
Codes of Conduct & 24 & 18.48 & 87 & 23.73 & -5.24 & 0.10 \\
Integrity Assurance & 24 & 31.60 & 87 & 31.70 & -0.11 & 0.99 \\
\hline Equal-weighted Total & 24 & 39.77 & 87 & 36.99 & 2.78 & 0.31 \\
Total excluding Governance & 24 & 36.12 & 87 & 38.05 & -1.93 & 0.48
\end{tabular}


Table 4 - Tests of differences in average scores between integrated and standalone reports: East Asia

\begin{tabular}{lll|ll|cc} 
& \multicolumn{2}{c|}{ Integrated } & \multicolumn{2}{c}{ Standalone } \\
\cline { 2 - 7 } & $\mathrm{N}$ & Mean & $\mathrm{N}$ & Mean & Difference & $\mathrm{p}$-value \\
\hline Chair's Message & 18 & 57.32 & 76 & 51.85 & 5.47 & 0.10 \\
Environment & 18 & 17.88 & 76 & 33.91 & -16.03 & 0.00 \\
Philanthropy & 18 & 44.44 & 76 & 55.70 & -11.26 & 0.09 \\
Stakeholder Engagement & 18 & 23.02 & 76 & 34.07 & -11.05 & 0.05 \\
Supply Chain Management & 18 & 15.20 & 76 & 34.48 & -19.29 & 0.00 \\
Labor Relations & 18 & 26.91 & 76 & 44.04 & -17.13 & 0.00 \\
Governance & 18 & 68.89 & 76 & 36.32 & 32.57 & 0.00 \\
Anti-corruption & 18 & 15.93 & 76 & 30.09 & -14.16 & 0.01 \\
Human Rights & 18 & 23.53 & 76 & 31.73 & -8.20 & 0.25 \\
Codes of Conduct & 18 & 13.36 & 76 & 19.05 & -5.69 & 0.03 \\
Integrity Assurance & 18 & 12.96 & 76 & 30.26 & -17.30 & 0.01 \\
\hline Equal-weighted Total & 18 & 29.04 & 76 & 36.50 & -7.46 & 0.01 \\
Total excluding Governance & 18 & 25.05 & 76 & 36.52 & -11.46 & 0.00
\end{tabular}




\section{Appendix}

List of CSR-S Monitor Contextual Elements

\begin{tabular}{|cl|}
\hline CSR-S Monitor Contextual Elements \\
\hline$\circ$ & Chair's / Executive Message (CHR) \\
$\circ$ & Environment (ENV) \\
$\circ$ & Philanthropy (PHL) \\
$\circ$ & Stakeholder Engagement (STK) \\
$\circ$ & Supply Chain Management (SCM) \\
$\circ$ & Labor Relations (LBR) \\
$\circ$ & Governance (GOV) \\
$\circ$ & Anti-corruption (COR) \\
$\circ$ & Human Rights (HR) \\
$\circ$ & Codes of Conduct: (COD) \\
& $\circ \quad$ Company codes \\
& $\circ \quad$ Industry-wide codes \\
& \\
&
\end{tabular}

Source: www.csrsmonitor.org 